\title{
Oral ingestion of the environmental toxicant trichlorethylene in rats induces alterations in the gut microbiome: relevance to idiopathic Parkinson's disease
}

\author{
Neda M. Ilieva, Zachary D. Wallen, Briana R. De Miranda* \\ Center for Neurodegeneration and Experimental Therapeutics, Department of Neurology, University of Alabama at Birmingham, \\ Birmingham, AL, USA
}

Microbial alterations within the gut microbiome appear to be a common feature of individuals with Parkinson's disease (PD), providing further evidence for the role of the gut-brain axis in PD development. As a major site of contact with the environment, questions have emerged surrounding the cause and effect of alterations to the gut microbiome by environmental contaminants associated with PD risk, such as pesticides, metals, and organic solvents. Recent data from our lab shows that ingestion of the industrial byproduct and environmental pollutant trichloroethylene (TCE) induces key Parkinsonian pathology within aged rats, including the degeneration of dopaminergic neurons, $\alpha$-synuclein accumulation, neuroinflammation, and endolysosomal deficits. As TCE is the most common organic contaminant within drinking water, we postulated that ingestion of TCE associated with PD-related neurodegeneration may alter the gut microbiome to a similar extent as observed in persons with PD. To assess this, we collected fecal samples from adult rats treated with $200 \mathrm{mg} / \mathrm{kg}$ TCE over 6 weeks via oral gavage and analyzed the gut microbiome via whole genome shotgun sequencing. Our results showed changes in gut microorganisms reflective of the microbial signatures observed in individuals with idiopathic PD, such as decreased abundance of short-chain fatty acid producing Blautia and elevated lactic-acid producing Bifidobacteria, as well as genera who contain species previously reported as opportunistic pathogens such as Clostridium. From these experimental data, we postulate that TCE exposure within contaminated drinking water could induce alterations of the gut microbiome that contributes to chronic disease risk, including idiopathic PD.

\section{Introduction}

Trichloroethylene (TCE) and structurally similar chlorinated solvents are pervasive environmental toxicants that contaminate air, water, and soil throughout the world (Bonvallot N 2010; Cancer 2014; Liu et al. 2020). Restrictions on TCE use within the United States and Europe have reduced its overall effluence into the environment in recent years, however the US Agency for Toxic Substances and Disease Registry (ATSDR) reports that TCE is the most frequently reported organic groundwater contaminant in the US, estimated to be present in 9 to $34 \%$ of drinking water (Todd et al. 2019). In addition, use of TCE as a degreasing agent in food production

\footnotetext{
*Correspondence should be addressed to

Briana R. De Miranda

University of Alabama at Birmingham

$17196^{\text {th }}$ Ave South | CIRC 516 | Birmingham, AL 35294

Tel: 205-996-2138

Email: BrianaDeMiranda@uabmc.edu
}

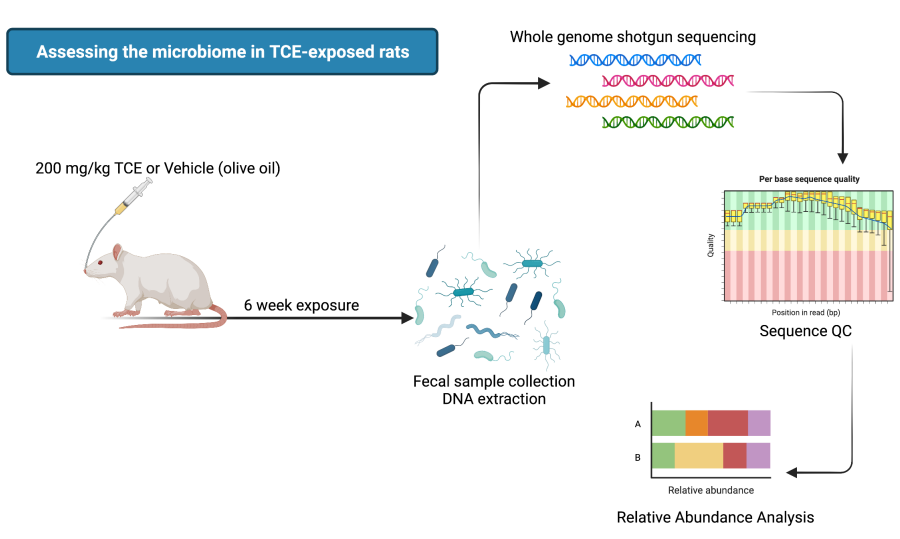

machinery results in contamination of certain processed foods, the most significant of which are butter and margarine, which averaged $73.6 \mu \mathrm{g} / \mathrm{kg}$ when sampled in 1995 (USEPA 2001). Despite the reduction of TCE usage in some nations, production of TCE remains at a steady global growth, resulting in reports of heavily contaminated drinking water as recently as 2020 within certain global regions, such as the Lanzhou district of China (Liu et al. 2020). Because TCE is 
environmentally persistent, it will continue to be a global contaminant of concern for decades to come.

As risk for chlorinated solvent ingestion remains in place, questions about the effect on the gut microbiome and its role in chronic disease have arisen. TCE exposure is associated with autoimmune diseases, such as systemic lupus erythematosus and autoimmune hepatitis, which are linked to altered gut microbiome composition (Anagnostopoulos et al. 2004; Kamijima et al. 2007). Experimental models of autoimmune disease from TCE exposure show that ingestion of low levels of the solvent within drinking water (e.g., 0.5 $\mathrm{mg} / \mathrm{mL}$ ) resulted in gut microbiome alterations such as increased abundance of Akkermansiaceae and Lachnospiraceae families (Hui Wang et al. 2021). In addition, TCE-induced microbiome changes were associated with altered gut cytokine levels, elevated colonic oxidative stress, and decreased intestinal epithelial tight junction protein expression (Blossom et al. 2020; Khare et al. 2019; Hui Wang et al. 2021).

Epidemiological evidence also links chlorinated solvent exposure, such as TCE, tetrachloroethylene (PERC), and methylene chloride, with elevated risk for Parkinson's disease (PD) (Goldman et al. 2012; Nielsen et al. 2021). Similarly, rodent studies of TCE exposure, including our own, recapitulate multiple features of PD pathogenesis, including the degeneration of nigrostriatal dopaminergic neurons (Liu et al. 2010; Liu et al. 2018), glial inflammation, endolysosomal deficits, and the accumulation of $\alpha$ synuclein (De Miranda et al. 2021). As increasing evidence supports a role for the gut microbiome in the complex etiology of environmental, lifestyle, and genetic factors that contribute to idiopathic PD (Romano et al. 2021; Toh et al. 2022; Wallen et al. 2020), we postulated that TCE ingestion may induce gut microbiome alterations that influence neurodegeneration.

Previously reported data from our lab shows that aged rats exposed to $200 \mathrm{mg} / \mathrm{kg}$ TCE via daily oral gavage (or control, olive oil) for six weeks results in selective degeneration of nigrostriatal dopaminergic neurons, elevated nigral oxidative stress, endolysosomal impairment, and a-synuclein accumulation in the substantia nigra (De Miranda et al. 2021). To assess the effect of TCE on the gut microbiome at the same dose and schedule that induces Parkinsonian neurodegenerative pathology, we treated aged adult, female Lewis rats with $200 \mathrm{mg} / \mathrm{kg}$ TCE or vehicle (olive oil) via oral gavage for six weeks and collected fecal samples for whole genome shotgun sequencing. The resulting metagenomic analysis showed that TCE ingestion induced significant alterations in the gut microbiome. Some of the microbial signatures detected mirrored those observed in individuals with idiopathic PD, such as decreased abundance in the butyrate producing genus Blautia, and elevated abundance of lactic-acid producing Bifidobacteria and genera who contain species previously reported as opportunistic pathogens such as Clostridium (Romano et al. 2021; Wallen et al. 2020). In addition, some species-level alterations detected by metagenomic sequencing in TCE exposed rats were reflective of reported changes in genera from PD gut microbiota, such as Parasutterella (Mao et al. 2021), Adlercreutzia (Zhang et al. 2020), and Mucispirillum (Lin et al. 2019). Collectively, these data suggest that TCE exposure associated with nigrostriatal Parkinsonian pathology, also alters the gut microbiome in rats with similarities observed in idiopathic PD. As an environmental contaminant with relevant ingestion risk, we suspect that chronic alteration of the gut microbiome by TCE exposure may influence PD etiology via the gut-brain axis.

\section{Materials and Methods}

\section{Reagents}

Standard laboratory reagents and chemicals were purchased from Millipore Sigma (Burlington, MA) or Thermo Fisher Scientific (Waltham, MA). Trichloroethylene was purchased through Thermo Fisher Scientific (CAS \#79-016). Premium olive oil was purchased at Trader Joes (Monrovia, CA).

\section{Animals and treatment}

Female Lewis rats aged 11 months were purchased through a retired breeding program at Envigo Biosciences (Indianapolis, IN) and transported to the University of Alabama at Birmingham small animal facility for a oneweek acclimation period prior to study onset. Rats were provided with standard rodent chow and filtered water $a d$ libitum throughout the study period. Following a one-week handling period, rats were given a daily oral gavage of 200 $\mathrm{mg} / \mathrm{kg}$ TCE or vehicle (olive oil) using flexible polypropylene gavage tubes (Instech, Plymouth Meeting, PA), 6-week period as reported in (De Miranda et al. 2021). At the study endpoint, rats were placed inside of sterilized 
microisolator cages where fecal matter was collected and immediately flash frozen and stored at $-80^{\circ} \mathrm{C}$. At the study termination, rats were humanely euthanized using a lethal dose of pentobarbital euthanasia, and underwent transcardial perfusion with PBS, followed by organ collection.

\section{DNA extraction, sequencing, and bioinformatics}

Frozen fecal samples were sent to CosmosID (Germantown, $\mathrm{MD}$ ) for DNA extraction and whole bacterial genome DNA (metagenome) shallow shotgun sequencing. DNA from samples was isolated using the QIAGEN DNeasy PowerSoil Pro Kit, according to the manufacturer's protocol. Samples were quantified using the GloMax Plate Reader System (Promega) using the QuantiFluor ${ }^{\circledR}$ dsDNA System (Promega). DNA libraries were prepared using the Nextera XT DNA Library Preparation Kit (Illumina) and IDT Unique Dual Indexes with total DNA input of 1ng. Genomic DNA was fragmented using a proportional amount of Illumina Nextera XT fragmentation enzyme. Unique dual indexes were added to each sample followed by 12 cycles of PCR to construct libraries. DNA libraries were purified using AMpure magnetic Beads (Beckman Coulter) and eluted in QIAGEN EB buffer. Libraries were then sequenced on an Illumina HiSeq X platform 2x150bp.

Unassembled sequencing reads were directly analyzed by CosmosID bioinformatics platform for multi-kingdom microbiome analysis and profiling of antibiotic resistance and virulence genes and quantification of organisms' relative abundance as described in (Hasan et al. 2014; Lax et al. 2014; Ottesen et al. 2016; Ponnusamy et al. 2016). Briefly, the system utilizes curated genome databases and a highperformance data-mining algorithm that rapidly disambiguates hundreds of millions of metagenomic sequence reads into the discrete microorganisms engendering the particular sequences. Similarly, the community resistome and virulome, the collection of antibiotic resistance and virulence associated genes in the microbiome, were also identified by querying the unassembled sequence reads against the CosmosID curated antibiotic resistance and virulence associated gene databases. Functional profiling and relative abundance from microbiome samples were identified using the strain-level curated CosmosID bacteria database, available through the CosmosID Bioinformatics Platform (CosmosID Metagenomics Cloud, app.cosmosid.com, CosmosID, Inc. www.cosmosid.com).

\section{Data analysis}

Statistical analyses were conducted using $\mathrm{R}$ Studio, GraphPad Prism 5.0 software (GraphPad Software Inc., San Diego, USA), and CosmosID Bioinformatics Platform as indicated. Principal Components Analysis, visualization of the PCA, and graphics were conducted using the
'FactoMineR' version 2.4 and 'factoextra' version 1.0.7 packages for R. Metagenomic sequences were filtered by the CosmosID Bioinformatics Platform using a filtering threshold that considers significant results based on internal statistical scores determined by analyzing a large number of diverse metagenomes. The resulting relative abundance data was log transformed. PCA was conducted on log transformed relative abundances of bacterial species, using the PCA function from 'FactoMineR' and visualized via the 'fviz_pca_ind' function from 'factoextra'. PCA grouping was based on the treatment group (VEH or TCE) and ellipses were created for the confidence intervals $(95 \% \mathrm{CI})$ of each group. Permutational ANOVA was conducted using the 'vegan' package in $\mathrm{R}$ version $2.5-7$ via the adonis function.

Shannon and Simpson indices for alpha-diversity were calculated using the 'diverse' package version 1.9.90 function 'diversity' in R for both VEH and TCE groups. Data was exported then plotted in GraphPad Prism Software. Statistical differences between untransformed relative abundance of bacterial phyla from VEH and TCE animals was determined using two-way ANOVA with a Sidak multiple comparison's post-hoc test (Graphpad Prism Software). Linear discriminant analysis (LDA) effect size (LEfSe) was used to ascertain taxonomic differences of untransformed metagenomic data of relative abundance of bacterial species from VEH and TCE groups, performed with the CosmosID Bioinformatics Platform. Only taxa with $\mathrm{p}<0.05$ (Kruskal-Wallis test) were compared for effect size using LDA, and features that reached LDA $>2$ were plotted as discriminatory features between treatment groups. Comparisons of untransformed metagenomic data of relative abundance within gut microbiome bacterial genera and species from VEH or TCE treated rats were conducted using an unpaired student's T-test using Graphpad Prism.

\section{Results}

\section{TCE ingestion influences gut microbiome composition}

Two-dimensional principal component analysis (PCA) based on log-transformed relative abundances of bacterial species revealed a separation between VEH and TCE treated rats. Differences in gut microbiome compositions observed in PCA were statistically significant $(p=0.008)$, tested using PERMANOVA (Figure 1a). Comparable species richness was observed in TCE and VEH groups measured with Simpson and Shannon indices (Figure 1b-c). Bacterial phyla of VEH and TCE animal gut microbiota with $1 \%$ or more relative abundance are depicted in Figure 2a, with no significant differences being detected between taxa $(p=0.77)$. At the genus level, TCE ingestion 
caused a marked elevation in Bifidobacterium $(p<$ $0.0001)$ and a reduction in Muribaculaceae $(p=0.25)$ and Ligilactobacillus $(p<0.0001)$ compared to VEH (Figure 2b-c). Using the linear discriminant analysis (LDA) effect size (LEfSe) analysis, we identified differential relative abundance in several features between VEH and TCE treated animals that were considered statistically significant with $p<0.05$ (Kruskal-Wallis test) and LDA score $>2$ (Figure 3).

\section{Microbial signatures in TCE treated rats share similarities with idiopathic PD}

We identified several trends in bacterial species from TCE treated rats that matched published reports of microbiome composition reported in individuals with idiopathic PD compared to healthy controls (Table 1). A decrease in the short-chain fatty acid (SCFA) producing bacteria Blautia was a prominent feature of TCE treated rat microbiome compared to VEH $(p<$ 0.05), which is consistently reported in idiopathic PD gut microbiota (Romano et al. 2021; Wallen et al. 2020), as well as other chronic diseases (Liu et al. 2021; Ren et al. 2020) (Figure 4a). Bacterial genera such as Clostridium $(p<0.05)$ and Bifidobacterium $(p<0.05)$, which are observed in greater abundance in the gut microbiota of PD patients (Sara Gerhardt and M. Hasan Mohajeri 2018; E. M. Hill-Burns et al. 2017; Romano et al. 2021; Wallen et al. 2020), were significantly increased in TCE treated animals compared to VEH (Figure 4b-d). A type of unclassified Burkholderiales species which belong to Burkholderia, a bacterial genus involved in generating hydrophobic bile acids (Wallner et al. 2019), displayed elevated abundance in TCE treated rats $(p<0.001)$, sharing a similar pattern with observations made from genera in the PD appendix (Li et al. 2021). Bacterial species Parasutterella excrementhominis $(p<0.001)$, Adlercreutzia equolifaciens $(p<0.05)$, and Muscipirillum schaedleri $(p<0.05)$, and Holdemania filiformis $(p<0.01)$ were also observed as significantly elevated in TCE treated rats which reflects what has been observed at the genus level from the PD gut microbiome (Lin et al. 2019; Mao et al. 2021; Zhang et al. 2020) (Figure 4e-h). A comparison of differentially abundant microbiota in TCE exposed rats (versus $\mathrm{VEH}$ ) with reported alterations in PD (versus health control) is described in Table 1.

\section{Functional profiling of biochemical activities from relative abundance}

Whole genome shotgun sequencing data was analyzed for functional biochemical activities using the MetaCyc Pathways database via the CosmosID Bioinformatics Platform. TCE exposed rats displayed significantly increased activity in pathways involving ATP synthesis and hydrolysis $(p=0.0086, p=0.0076$ compared to $\mathrm{VEH}$, respectively). Though limited data are available to compare the effect of TCE with human PD microbiome functional pathways, some reports of similarly altered biochemical processes in TCE treated rats, such as ubiquinone biosynthetic processes, were observed in idiopathic PD and MSA (Engen et al. 2017; Keshavarzian et al. 2015) (Figure 5).

\section{Discussion}

Exposure to environmental toxicants via ingestion may induce gut microbiome alterations that contribute to increased risk for chronic disease including idiopathic PD. Despite some variability in human population study data (Keshavarzian et al. 2020), a gut microbiome signature appears to be present in most individuals with PD that includes the depletion in relative abundance of SCFA producing genera, such as Blautia, and increased relative abundance of Bifidobacteria, Akkermansia, Clostridium, and Lactobacillus (Heinzel et al. 2021; E. M. Hill-Burns et al. 2017; Toh et al. 2022; Wallen et al. 2020). Thus, a convincing explaination suggests that altered gut microbiota contribute to elevated GI and systemic inflammation that may drive neuroinflammatory changes and/or influence the aggregation and spread of $\alpha$-synuclein along the gut-brain axis (Houser and Tansey 2017). In addition, the observed gut microbiome alterations in PD also indicate a role for environmental exposures that increase risk for the disease, such as pesticides, metals, air pollution, organic solvents, and pathogens, as the GI tract represents the most profuse site of environmental contact within the body (Keshavarzian et al. 2020).

At a dose shown to produce dopaminergic neurodegeneration from the nigrostriatal tract, exposure to $200 \mathrm{mg} / \mathrm{kg}$ TCE over 6 weeks in adult rats resulted in a profound dysbiosis in the gut microbiome, with a high degree of concordance to reported 
bioRxiv preprint doi: https://doi.org/10.1101/2022.02.19.481161; this version posted February 20, 2022. The copyright holder for this preprint (which was not certified by peer review) is the author/funder. All rights reserved. No reuse allowed without permission.

\begin{tabular}{|c|c|c|c|}
\hline \multirow{2}{*}{ Bacterial Species } & \multirow{2}{*}{$\begin{array}{c}\text { TCE } \\
\text { Microbiome }\end{array}$} & \multicolumn{2}{|c|}{ Abundance in Parkinson's Patients (Associated Genus or Species) } \\
\hline & & Increased & Reduced \\
\hline Blautia wexlerae & Reduced & $\begin{array}{l}\text { 个 (Heintz-Buschart et al. 2018; Li et } \\
\text { al. 2019; Scheperjans et al. 2015) }\end{array}$ & $\begin{array}{l}\downarrow \text { (Aho et al. 2019; Barichella et al. } \\
\text { 2019; E. M. Hill-Burns et al. 2017; } \\
\text { Petrov et al. 2017; Takahashi et al. } \\
\text { 2022; Zapała et al. 2021) }\end{array}$ \\
\hline Clostridium (uc) & Increased & $\begin{array}{l}\text { 个 (Heintz-Buschart et al. 2018; } \\
\text { Kang et al. 2020; Keshavarzian et } \\
\text { al. 2015; Lin et al. 2018; Lubomski } \\
\text { et al. 2020; Murros 2021; Murros et } \\
\text { al. 2021; Qian et al. 2018; Romano } \\
\text { et al. 2021) }\end{array}$ & $\begin{array}{l}\downarrow \text { (Aho et al. 2019; Bedarf et al. 2017; } \\
\text { Hasegawa et al. 2015) }\end{array}$ \\
\hline $\begin{array}{l}\text { Bifidobacterium } \\
\text { pseudolongum }\end{array}$ & Increased & $\begin{array}{l}\text { 个 (Barichella et al. 2019; Heintz- } \\
\text { Buschart et al. 2018; Erin M. Hill- } \\
\text { Burns et al. 2017; Lin et al. 2018; } \\
\text { Petrov et al. 2017; Romano et al. } \\
\text { 2021; Unger et al. 2016) }\end{array}$ & \\
\hline $\begin{array}{l}\text { Burkholderiales } \\
\text { (uc) }\end{array}$ & Increased & 个 (Peipei Li et al. 2020) & \\
\hline $\begin{array}{c}\text { Parasutterella } \\
\text { excrementihominis }\end{array}$ & Increased & $\begin{array}{l}\text { 个 (Heintz-Buschart et al. 2018; } \\
\text { Heinzel et al. 2021; Mao et al. 2021; } \\
\text { Tan et al. 2018) }\end{array}$ & $\downarrow$ (Erin M. Hill-Burns et al. 2017) \\
\hline $\begin{array}{l}\text { Adlercreutzia } \\
\text { eqolifaciens }\end{array}$ & Increased & $\begin{array}{l}\uparrow(\text { Jin et al. 2019; Zhang et al. } \\
2020)\end{array}$ & \\
\hline $\begin{array}{l}\text { Mucispirillum } \\
\text { schaedleri }\end{array}$ & Increased & $\begin{array}{l}\uparrow(\text { Lin et al. 2019; Rajput et al. } \\
2021)\end{array}$ & \\
\hline $\begin{array}{l}\text { Akkermansia } \\
\text { muciniphila }\end{array}$ & Reduced & $\begin{array}{l}\text { 个 (Barichella et al. 2019; Bedarf et } \\
\text { al. 2017; Heintz-Buschart et al. } \\
\text { 2018; Erin M. Hill-Burns et al. 2017; } \\
\text { Keshavarzian et al. 2015; Li et al. } \\
\text { 2019; Romano et al. 2021; Unger et } \\
\text { al. 2016) }\end{array}$ & $\downarrow \quad$ (Aho et al. 2019) \\
\hline $\begin{array}{l}\text { Ligilactobacillus } \\
\text { animalis/murinus }\end{array}$ & Reduced & $\begin{array}{l}\uparrow \quad \text { (Aizawa et al. 2019; Romano et } \\
\text { al. 2021) }\end{array}$ & \\
\hline $\begin{array}{l}\text { Holdemania } \\
\text { filiformis }\end{array}$ & Increased & $\begin{array}{l}\text { 个 (Mao et al. 2021; Qian et al. } \\
2018)\end{array}$ & \\
\hline
\end{tabular}

Table 1. Differentially abundant bacteria from TCE treated rats in relation to microbiota in persons with PD. Species data are reported from metagenomic analyses in TCE treated rats belonging to genera in 16S rRNA or targeted gene sequencing from human data. Unclassified species belonging to a genera are labed as (uc).

microbiota from idiopathic PD. The strong reduction in abundance of the SCFA producing genus Blautia from TCE-exposed rats is a similar feature to PD, as well as other chronic diseases, such as inflammatory bowel disease and ulcerative colitis, both of which are GI disorders associated with PD development (Villumsen et al. 2019). Conversely, significantly increased abundance of Bifidobacterium, Clostridium, and Burkholderiales was observed in TCE treated rats, which have been reported in one or more metagenomic or $16 \mathrm{~S}$ datasets comparing the gut microbiome of healthy controls to individuals with PD (Keshavarzian et al. 2015; Keshavarzian et al. 2020; Li et al. 2021; Wallen et al. 2020). These data imply that similarities in the PD gut microbiome can be at least partially reproduced by exposure to a single environmental toxicant associated with elevated risk for the disease.

TCE is a highly lipophilic small molecule, which readily crosses biological membranes and is 
extensively metabolized by the liver and kidneys (Cichocki et al. 2016; Lash et al. 2006). Absorption of TCE or similar chlorinated solvents within the GI tract therefore may directly damage microorganisms and/or intestinal epithelial cells and proteins, resulting in elevated systemic inflammation that may ultimately influence PD development (Houser and Tansey 2017; Khan et al. 2009). Epidemiological evidence for GI damage from chlorinated solvent exposure exists; for example, individuals who were exposed to public drinking water contaminated with tetrachloroethylene (PERC) had higher risk for colorectal cancer (Paulu et al. 1999). In addition, the association of TCE with autoimmune disease (Phillips et al. 2019) points to an intersection of GI pathology and microbiome dysbiosis from ingestion exposure that could influence inflammatory bowel diseases. Animal studies in the autoimmune mouse line $\mathrm{MRL}^{+/+}$showed that exposure to TCE via drinking water resulted in irreversible gut microbiome dysbiosis, even after exposure cessation, specifically elevated Bifidobacterium, the genus found to be elevated to the greatest extent in this study (Khare et al. 2019). A separate study of TCE exposure in $\mathrm{MRL}^{+/+}$mice showed TCE ingestion resulted in decreased expression of tight junction proteins $\mathrm{ZO}-2$, occludin, and claudin-3, as well as elevated proinflammatory proteins CD 14 and IL- $1 \beta$ in the colon (Hui Wang et al. 2021). Together, these data suggest that TCE ingestion induces a proinflammatory environment within the gut, with a possible bidirectional interplay between gut microbes, immune regulation, and intestinal pathology.

Though gut microbiome alteration is linked with TCE ingestion in animal models, it remains unclear what the functional implications of bacterial abundance mean in the context of environmental risk for PD. In the environment, TCE is metabolized by soil microorganisms, predominantly through reductive dichlorination by anaerobic bacteria (Freedman and Gossett 1989). In soil contaminated by TCE, microbiota exhibit inhibited energy metabolism and a reduction in the metabolic capacity for other xenobiotics (P. Li et al. 2020; Moccia et al. 2017). Thus, in addition to inflammatory promoting factors in the gut, TCE exposure could influence xenobiotic metabolism by gut microbiota, potentially resulting in altered enterohepatic cycling, host cytochrome P450 metabolizing enzyme expression, and reduced detoxification or elevated bioactivation. Interestingly, gut microbiota from TCE exposed rats displayed significantly increased activity in pathways involving ATP synthesis and hydrolysis, possibly related to elevated P450 metabolism (Foti and Dalvie 2016). Gut microbiota from individuals with PD displayed elevated xenobiotic degradation pathways related to environmental contaminants such as pesticides and chloroalkanes (E. M. Hill-Burns et al. 2017). In concordance with this, we observed elevated abundance of Burkholderia in TCE treated rats, a genus which contains species that specifically degrade polychlorinated biphenyls (Tehrani et al. 2012), and were reported to be increased in the PD appendix ( $\mathrm{Li}$ et al. 2021). Collectively, these data support a role for environmental exposures potentially driving gut microbiome alterations observed in PD.

In line with the many other published studies investigating gut microbiome and $\mathrm{PD}$ related neurodegeneration, the data collected from TCE treated rats partially conflicted with human $\mathrm{PD}$ microbiome and other animal model studies (Keshavarzian et al. 2020). One of the major differences observed was the decrease in Akkermansia and Lactobacillus genera from TCE treated rats, compared to the relatively common increase found in human PD studies (E. M. Hill-Burns et al. 2017; Romano et al. 2021; Wallen et al. 2020; Zapała et al. 2021), though at least one or more studies report decreases in either genus ( $\mathrm{Li}$ et al. 2019; Qian et al. 2018). While the most obvious source of variation is likely rodent to human translation, $\mathrm{MRL}^{+/+}$mice exposed to TCE in drinking water displayed a significant elevation in Akkermansia within the gut microbiome compared to control (Hui Wang et al. 2021). In addition, TCE exposed $\mathrm{MRL}^{+/+}$mice displayed an apparent dose-dependent response in Lactobacillus abundance within the gut microbiome, as low dose TCE exposure resulted in increased Lactobacillus expression, however high dose TCE exposure was associated with its decrease after 259 days of exposure (Khare et al. 2019). As $200 \mathrm{mg} / \mathrm{kg}$ TCE in adult rats was a relatively high amount of TCE, reduced Lactobacillus abundance may be a result of dose-specificity. In relation, as the two autoimmune studies show, elevated Akkermansia abundance induced by TCE exposure could be a result of hostmicrobiome-environment interaction involving autoimmunity (Khare et al. 2019; H. Wang et al. 2021).

In addition to the disparate findings in certain genera, there were other limitations of this study. First, direct comparison of rats exposed to TCE and human PD is 
difficult; the inbred Lewis rats exposed in this study were fed a standard rodent chow and only underwent treatment with a single toxicant exposure over 6 weeks. In contrast, the microbiome from individuals with PD is influenced by diet, medications, location of residence, and simultaneous or previous exposure to multiple environmental factors (S. Gerhardt and M. H. Mohajeri 2018; E. M. Hill-Burns et al. 2017; Keshavarzian et al. 2020; Romano et al. 2021). In addition, complex gene-environment interactions that occur in individuals with PD were not available to measure in wildtype rats. Finally, many comparisons were made between $16 \mathrm{~S}$ sequencing reported in the literature and whole genome shallow shotgun sequencing performed here, possibly resulting in differences between curated microbiome databases and taxonomic resolution. Perhaps because of this, the observed similarities in gut microbiota of rats exposed to TCE with reports from individuals with PD is even more remarkable and indicates a strong connection between the gut microbiome and relevant environmental contaminants associated with PD risk. Future studies to assess the influence of the microbiome on the chronic neurotoxic properties of TCE will be essential in understanding PD risk from this ubiquitous toxicant.

\author{
Acknowledgments \\ This work was supported by research grants from the \\ National Institutes of Environmental Health Sciences \\ (R00ES029986, BRD), and the Parkinson Association of \\ Alabama (BRD).
}


A.

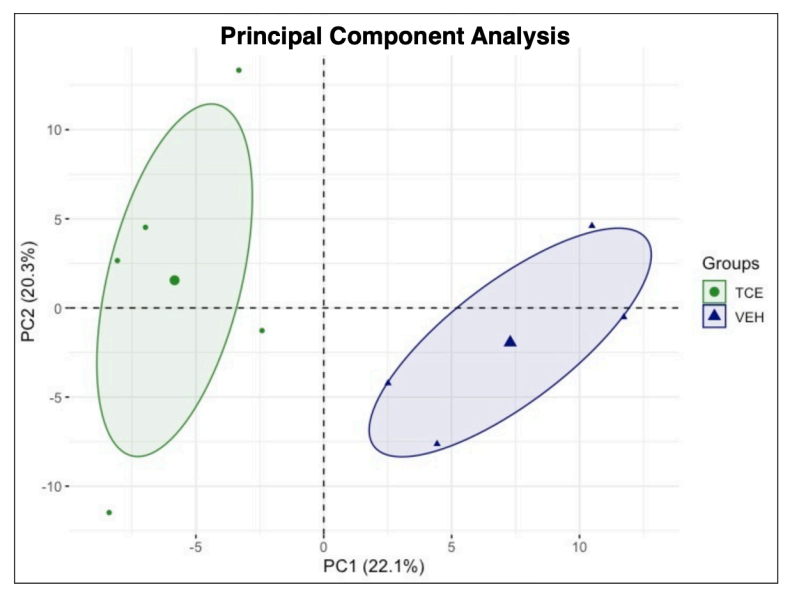

B.

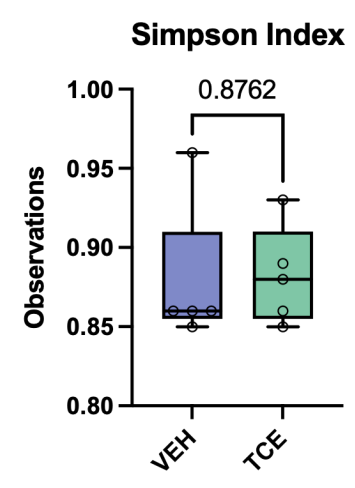

c.

Shannon Index

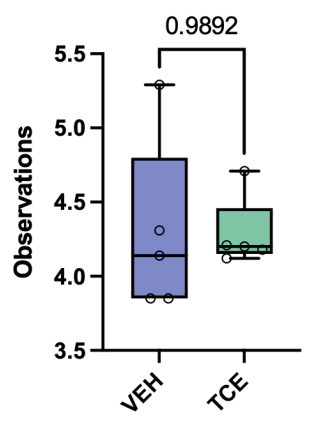

Figure 1. Gut microbiome composition measured by relative abundance differs between animals exposed to TCE and VEH. A. 2-Dimensional Principal Component Analysis (PCA) of relative abundances for all bacterial species sequenced, shows separation and independent clustering of TCE (green) and VEH (blue) groups, $\mathrm{p}=0.008$ (PERMANOVA). B-C. Simpson and Shannon indices demonstrate comparable diversity between microbiota from TCE and VEH animals. $\mathrm{N}=5$, box and whisker lines denote median and interquartile range. 

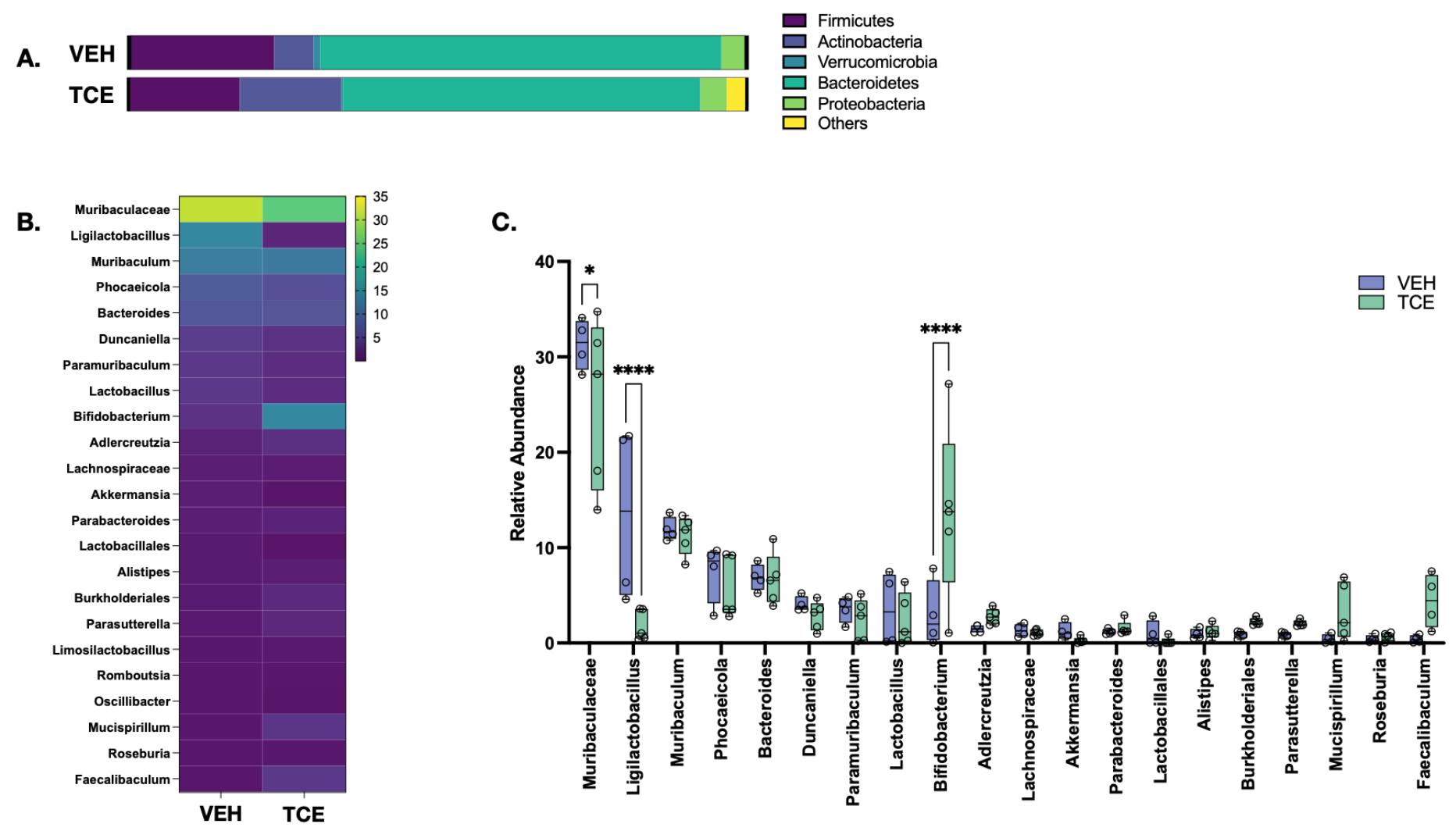

Figure 2. Relative abundance of gut microbiome genera is influenced by TCE exposure in rats.

A. Graphical representation of the relative abundances of phyla in VEH and TCE groups, with no statistical differences between taxa. B. Heatmap depiction of relative abundance of major gut microorganisms from VEH and TCE treated rats, scale bar indicates \% relative abundance. C. A comparison between relative abundance of bacterial genera from the microbiome of VEH and TCE rats with significantly decreased Muribaculaceae $(\mathrm{p}=0.025)$ and Ligilactobacillus $(\mathrm{p}=0.0001)$, and increased Bifidobacterium $(\mathrm{p}=$ 0.0001); $\mathrm{N}=5$, two-way ANOVA, ${ }^{*} p<0.05, * * * * p<0.0001$, box and whisker lines denote median and interquartile range. 


\section{Relative Abundance: Bacterial Species}

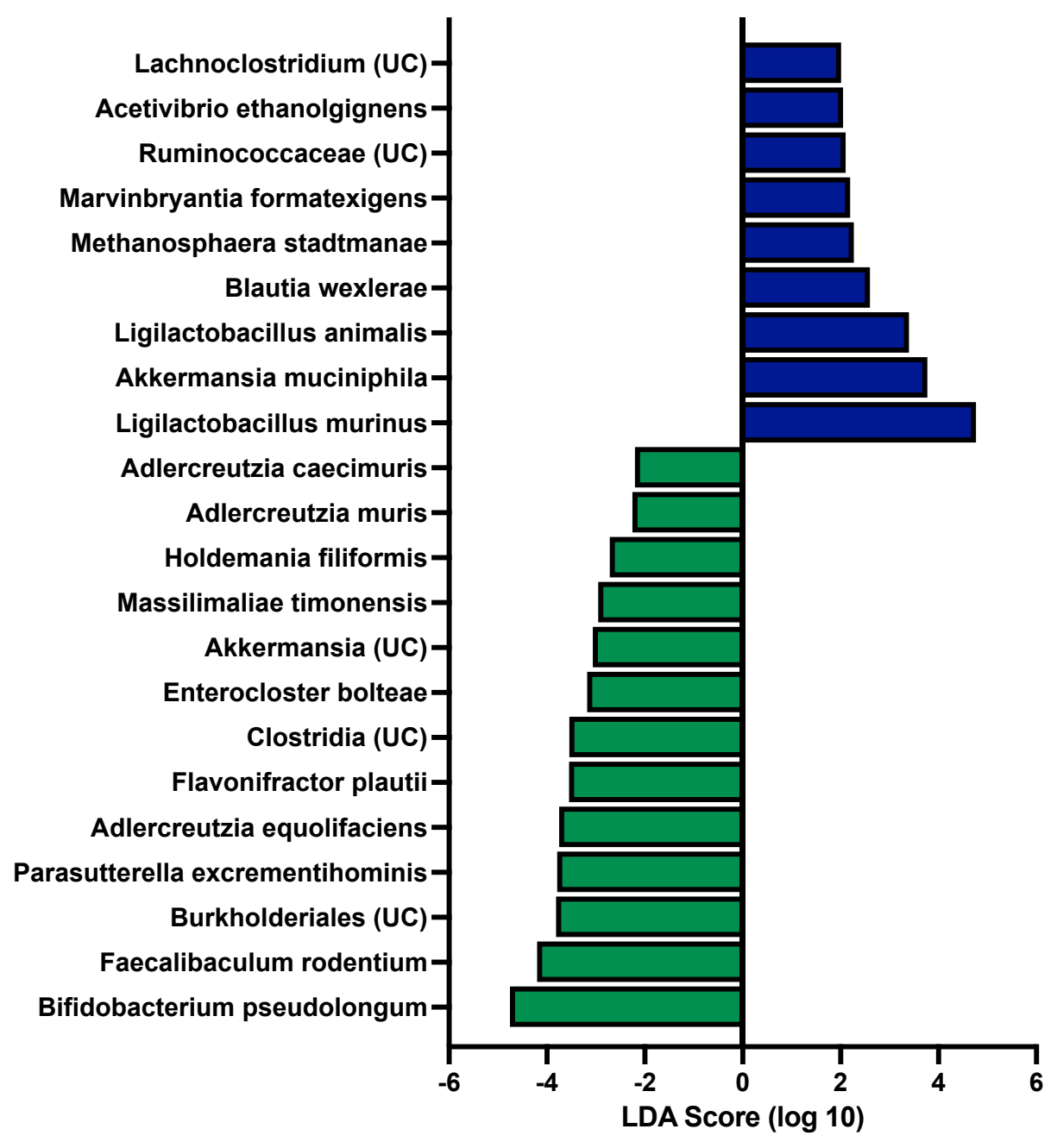

Figure 3. TCE ingestion resulted in significant alteration of gut microbiome bacterial species relative abundance. Linear discriminant analysis effect size (LEfSe) demonstrates relative abundance differences between metagenomic sequences of bacterial species from VEH (blue) and TCE (green) treated rats. Unclassified species of a bacterial genus are denoted as (UC). $p<0.05$ LDA score $>2, \mathrm{~N}=5$. 
A. Blautia wexlerae

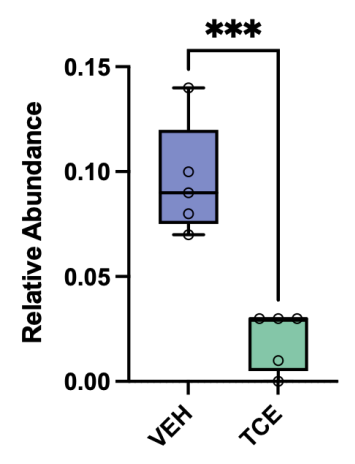

E. Parasutterella excrementihominis

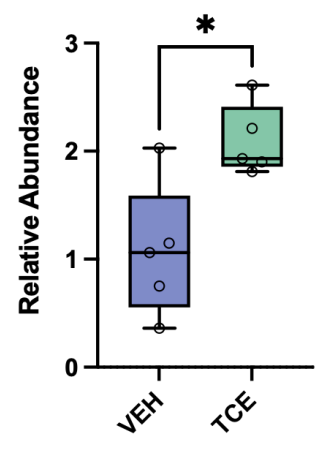

B. Clostridia (unclassified species)

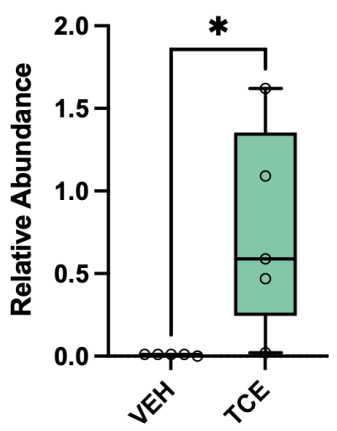

F. Adlercreutzia

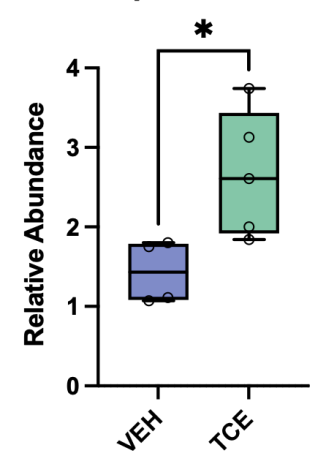

C. Bifidobacterium

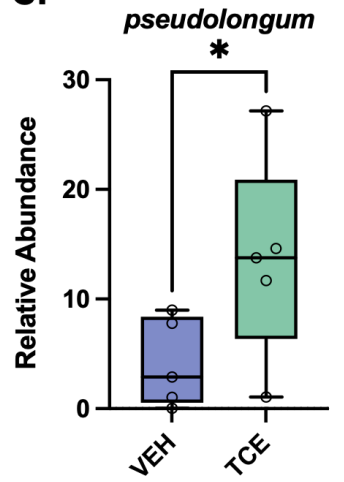

G. Mucispirillum schaedleri

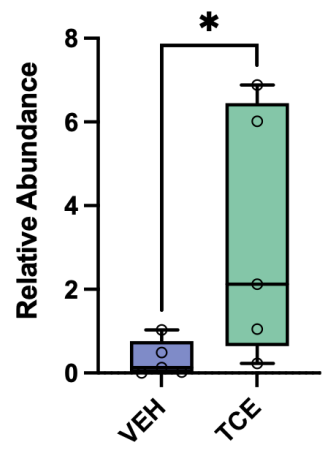

D. Burkholderiales (unclassified species)

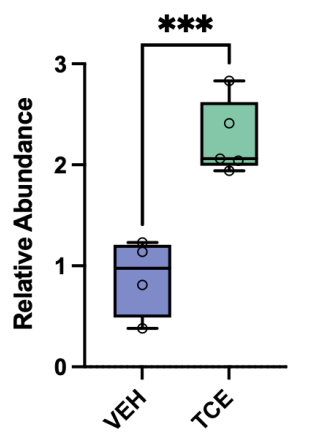

H. Holdemania filiformis

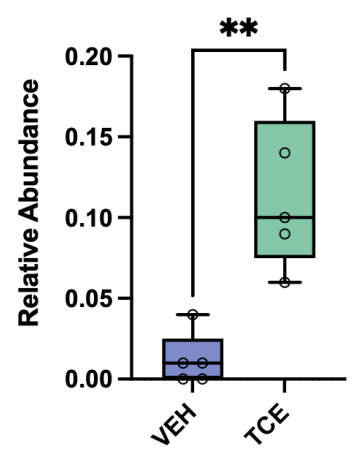

Figure 4. Relative abundance of gut microbial species within TCE-treated rats share similarities with alterations reported in PD. TCE treatment resulted in reduction of Blautia $(p=0.02 ; \mathbf{A})$, and greater relative abundance of an unspecified species of Clostridia $(p=0.025)$, Bifidobacterium $(p=0.031)$, and Burkholderiales species $(p=0.001)$, Parasutterella excrementihominis $(p=0.009)$, Adlercreutzia equolifaciens $(p=0.025)$, Mucispirillum schaedleri $(p=0.031)$, and Holdemania filiformis $(p=0.0017)$ compared to VEH animals, which are reported trends in PD gut microbiota (B-H). $\mathrm{N}=5, * p<0.05, * * p<0.01, * * * p<0.001$, box and whisker lines denote median and interquartile range. 


\section{Biological Processes}

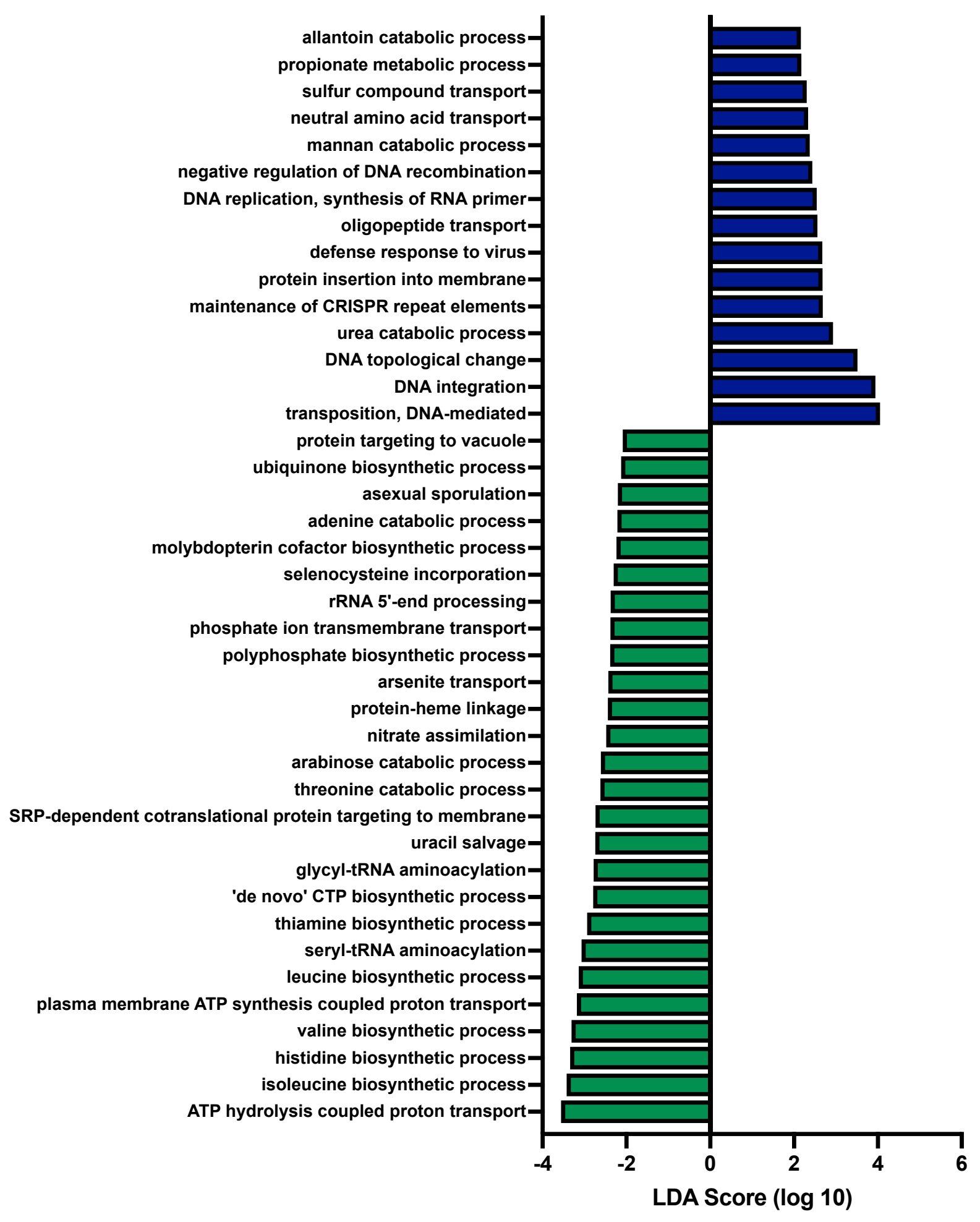

Figure 5. Predicted biological processes associated with TCE induced gut microbiome dysbiosis. LEfSe plot from pathway analysis data of biological processes (MetaCyC) shows significant differences in microbial biological processes between TCE and VEH rats. $p<0.05$ LDA score $>2, \mathrm{~N}=5$. 


\section{References}

Aho VTE, Pereira PAB, Voutilainen S, Paulin L, Pekkonen E, Auvinen P, et al. 2019. Gut microbiota in parkinson's disease: Temporal stability and relations to disease progression. EBioMedicine 44:691-707.

Aizawa E, Tsuji H, Asahara T, Takahashi T, Teraishi T, Yoshida S, et al. 2019. Bifidobacterium and lactobacillus counts in the gut microbiota of patients with bipolar disorder and healthy controls. Front Psychiatry 9:730-730.

Anagnostopoulos G, Sakorafas G, Grigoriadis K, Margantinis G, Kostopoulos P, Tsiakos S, et al. 2004. Hepatitis caused by occupational chronic exposure to trichloroethylene. Acta gastro-enterologica belgica 67:355-357.

Barichella M, Severgnini M, Cilia R, Cassani E, Bolliri C, Caronni S, et al. 2019. Unraveling gut microbiota in parkinson's disease and atypical parkinsonism. Movement Disorders 34:396-405.

Bedarf JR, Hildebrand F, Coelho LP, Sunagawa S, Bahram M, Goeser F, et al. 2017. Functional implications of microbial and viral gut metagenome changes in early stage 1-dopa-naïve parkinson's disease patients. Genome Medicine 9:39.

Blossom SJ, Gokulan K, Arnold M, Khare S. 2020. Sex-dependent effects on liver inflammation and gut microbial dysbiosis after continuous developmental exposure to trichloroethylene in autoimmune-prone mice. Front Pharmacol 11:569008.

Bonvallot N HP, Loh M. 2010. Trichloroethylene. In: Who guidelines for indoor air quality: Selected pollutants. Geneva: World health organization.

Cancer LFIAfRo. 2014. Iarc working group on the evaluation of carcinogenic risks to humans. Trichloroethylene, tetrachloroethylene, and some other chlorinated agents. (IARC Monographs on the Evaluation of Carcinogenic Risks to Humans).

Cichocki JA, Guyton KZ, Guha N, Chiu WA, Rusyn I, Lash LH. 2016. Target organ metabolism, toxicity, and mechanisms of trichloroethylene and perchloroethylene: Key similarities, differences, and data gaps. J Pharmacol Exp Ther 359:110-123.

De Miranda BR, Castro SL, Rocha EM, Bodle CR, Johnson KE, Greenamyre JT. 2021. The industrial solvent trichloroethylene induces lrrk2 kinase activity and dopaminergic neurodegeneration in a rat model of parkinson's disease. Neurobiol Dis 153:105312.

Engen PA, Dodiya HB, Naqib A, Forsyth CB, Green SJ, Voigt RM, et al. 2017. The potential role of gutderived inflammation in multiple system atrophy. J Parkinsons Dis 7:331-346.

Foti RS, Dalvie DK. 2016. Cytochrome p450 and non-cytochrome p450 oxidative metabolism: Contributions to the pharmacokinetics, safety, and efficacy of xenobiotics. Drug Metabolism and Disposition 44:1229-1245.

Freedman DL, Gossett JM. 1989. Biological reductive dechlorination of tetrachloroethylene and trichloroethylene to ethylene under methanogenic conditions. Appl Environ Microbiol 55:2144-2151.

Gerhardt S, Mohajeri MH. 2018. Changes of colonic bacterial composition in parkinson's disease and other neurodegenerative diseases. Nutrients 10.

Gerhardt S, Mohajeri MH. 2018. Changes of colonic bacterial composition in parkinson's disease and other neurodegenerative diseases. Nutrients 10:708.

Goldman SM, Quinlan PJ, Ross GW, Marras C, Meng C, Bhudhikanok GS, et al. 2012. Solvent exposures and parkinson disease risk in twins. Ann Neurol 71:776-784.

Hasan NA, Young BA, Minard-Smith AT, Saeed K, Li H, Heizer EM, et al. 2014. Microbial community profiling of human saliva using shotgun metagenomic sequencing. PLoS One 9:e97699.

Hasegawa S, Goto S, Tsuji H, Okuno T, Asahara T, Nomoto K, et al. 2015. Intestinal dysbiosis and lowered serum lipopolysaccharide-binding protein in parkinson's disease. PLOS ONE 10:e0142164.

Heintz-Buschart A, Pandey U, Wicke T, Sixel-Döring F, Janzen A, Sittig-Wiegand E, et al. 2018. The nasal and gut microbiome in parkinson's disease and idiopathic rapid eye movement sleep behavior disorder. Mov Disord 33:88-98.

Heinzel S, Aho VTE, Suenkel U, von Thaler A-K, Schulte C, Deuschle C, et al. 2021. Gut microbiome signatures of risk and prodromal markers of parkinson disease. Annals of Neurology 90:E1-E12. 
Hill-Burns EM, Debelius JW, Morton JT, Wissemann WT, Lewis MR, Wallen ZD, et al. 2017. Parkinson's disease and parkinson's disease medications have distinct signatures of the gut microbiome. Mov Disord 32:739-749.

Hill-Burns EM, Debelius JW, Morton JT, Wissemann WT, Lewis MR, Wallen ZD, et al. 2017. Parkinson's disease and parkinson's disease medications have distinct signatures of the gut microbiome. Movement Disorders 32:739-749.

Houser MC, Tansey MG. 2017. The gut-brain axis: Is intestinal inflammation a silent driver of parkinson's disease pathogenesis? npj Parkinson's Disease 3:3.

Jin M, Li J, Liu F, Lyu N, Wang K, Wang L, et al. 2019. Analysis of the gut microflora in patients with parkinson's disease. Front Neurosci 13:1184-1184.

Kamijima M, Hisanaga N, Wang H, Nakajima T. 2007. Occupational trichloroethylene exposure as a cause of idiosyncratic generalized skin disorders and accompanying hepatitis similar to drug hypersensitivities. Int Arch Occup Environ Health 80:357-370.

Kang X, Ploner A, Ludvigsson JF, Williams DM, Larsson H, Pedersen NL, et al. 2020. Clostridium difficile infection and risk of parkinson's disease: A swedish population-based cohort study. European Journal of Neurology 27:2134-2141.

Keshavarzian A, Green SJ, Engen PA, Voigt RM, Naqib A, Forsyth CB, et al. 2015. Colonic bacterial composition in parkinson's disease. Mov Disord 30:1351-1360.

Keshavarzian A, Engen P, Bonvegna S, Cilia R. 2020. Chapter 11 - the gut microbiome in parkinson's disease: A culprit or a bystander? In: Progress in brain research, Vol. 252, (Björklund A, Cenci MA, eds):Elsevier, 357-450.

Khan S, Priyamvada S, Khan SA, Khan W, Farooq N, Khan F, et al. 2009. Effect of trichloroethylene (tce) toxicity on the enzymes of carbohydrate metabolism, brush border membrane and oxidative stress in kidney and other rat tissues. Food and Chemical Toxicology 47:1562-1568.

Khare S, Gokulan K, Williams K, Bai S, Gilbert KM, Blossom SJ. 2019. Irreversible effects of trichloroethylene on the gut microbial community and gut-associated immune responses in autoimmuneprone mice. J Appl Toxicol 39:209-220.

Lash LH, Putt DA, Parker JC. 2006. Metabolism and tissue distribution of orally administered trichloroethylene in male and female rats: Identification of glutathione- and cytochrome p-450-derived metabolites in liver, kidney, blood, and urine. J Toxicol Environ Health A 69:1285-1309.

Lax S, Smith DP, Hampton-Marcell J, Owens SM, Handley KM, Scott NM, et al. 2014. Longitudinal analysis of microbial interaction between humans and the indoor environment. Science 345:1048-1052.

Li C, Cui L, Yang Y, Miao J, Zhao X, Zhang J, et al. 2019. Gut microbiota differs between parkinson's disease patients and healthy controls in northeast china. Frontiers in Molecular Neuroscience 12.

Li P, Killinger BA, Beddows I, Ensink E, Yilmaz A, Lubben N, et al. 2020. Gut microbiome dysbiosis is associated with elevated toxic bile acids in parkinson's disease. bioRxiv:2020.2009.2026.279851.

Li P, Zhang Y, Meng Q, Liu Y, Tuyiringire D, Chen Z, et al. 2020. Trichloroethylene inhibits nitrogen transformation and microbial community structure in mollisol. Ecotoxicology 29:801-813.

Li P, Killinger BA, Ensink E, Beddows I, Yilmaz A, Lubben N, et al. 2021. Gut microbiota dysbiosis is associated with elevated bile acids in parkinson's disease. Metabolites 11:29.

Lin A, Zheng W, He Y, Tang W, Wei X, He R, et al. 2018. Gut microbiota in patients with parkinson's disease in southern china. Parkinsonism \& Related Disorders 53:82-88.

Lin C-H, Chen C-C, Chiang H-L, Liou J-M, Chang C-M, Lu T-P, et al. 2019. Altered gut microbiota and inflammatory cytokine responses in patients with parkinson's disease. Journal of Neuroinflammation $16: 129$.

Liu M, Choi DY, Hunter RL, Pandya JD, Cass WA, Sullivan PG, et al. 2010. Trichloroethylene induces dopaminergic neurodegeneration in fisher 344 rats. J Neurochem 112:773-783.

Liu M, Shin E-J, Dang D-K, Jin C-H, Lee PH, Jeong JH, et al. 2018. Trichloroethylene and parkinson's disease: Risk assessment. Molecular Neurobiology 55:6201-6214.

Liu X, Mao B, Gu J, Wu J, Cui S, Wang G, et al. 2021. Blautia-a new functional genus with potential probiotic properties? Gut Microbes 13:1-21. 
Liu Y, Hao S, Zhao X, Li X, Qiao X, Dionysiou DD, et al. 2020. Distribution characteristics and health risk assessment of volatile organic compounds in the groundwater of lanzhou city, china. Environmental Geochemistry and Health 42:3609-3622.

Lubomski M, Tan AH, Lim S-Y, Holmes AJ, Davis RL, Sue CM. 2020. Parkinson's disease and the gastrointestinal microbiome. Journal of Neurology 267:2507-2523.

Mao L, Zhang Y, Tian J, Sang M, Zhang G, Zhou Y, et al. 2021. Cross-sectional study on the gut microbiome of parkinson's disease patients in central china. Front Microbiol 12.

Moccia E, Intiso A, Cicatelli A, Proto A, Guarino F, Iannece P, et al. 2017. Use of zea mays 1. In phytoremediation of trichloroethylene. Environ Sci Pollut Res Int 24:11053-11060.

Murros KE. 2021. Sulfate reducing gut bacteria and parkinson's disease. European Journal of Neurology 28:e21-e21.

Murros KE, Huynh VA, Takala TM, Saris PEJ. 2021. Desulfovibrio bacteria are associated with parkinson's disease. Front Cell Infect Microbiol 11:652617-652617.

Nielsen SS, Warden MN, Sallmen M, Sainio M, Uuksulainen S, Checkoway H, et al. 2021. Solvent exposed occupations and risk of parkinson disease in finland. Clin Park Relat Disord 4:100092.

Ottesen A, Ramachandran P, Reed E, White JR, Hasan N, Subramanian P, et al. 2016. Enrichment dynamics of listeria monocytogenes and the associated microbiome from naturally contaminated ice cream linked to a listeriosis outbreak. BMC microbiology 16:275-275.

Paulu C, Aschengrau A, Ozonoff D. 1999. Tetrachloroethylene-contaminated drinking water in massachusetts and the risk of colon-rectum, lung, and other cancers. Environ Health Perspect 107:265-271.

Petrov VA, Saltykova IV, Zhukova IA, Alifirova VM, Zhukova NG, Dorofeeva YB, et al. 2017. Analysis of gut microbiota in patients with parkinson's disease. Bulletin of Experimental Biology and Medicine 162:734737.

Phillips RV, Rieswijk L, Hubbard AE, Vermeulen R, Zhang J, Hu W, et al. 2019. Human exposure to trichloroethylene is associated with increased variability of blood DNA methylation that is enriched in genes and pathways related to autoimmune disease and cancer. Epigenetics 14:1112-1124.

Ponnusamy D, Kozlova EV, Sha J, Erova TE, Azar SR, Fitts EC, et al. 2016. Cross-talk among flesh-eating aeromonas hydrophila strains in mixed infection leading to necrotizing fasciitis. Proc Natl Acad Sci U S A 113:722-727.

Qian Y, Yang X, Xu S, Wu C, Song Y, Qin N, et al. 2018. Alteration of the fecal microbiota in chinese patients with parkinson's disease. Brain, Behavior, and Immunity 70:194-202.

Rajput C, Sarkar A, Sachan N, Rawat N, Singh MP. 2021. Is gut dysbiosis an epicenter of parkinson's disease? Neurochemical Research 46:425-438.

Ren Z, Fan Y, Li A, Shen Q, Wu J, Ren L, et al. 2020. Alterations of the human gut microbiome in chronic kidney disease. Advanced Science 7:2001936.

Romano S, Savva GM, Bedarf JR, Charles IG, Hildebrand F, Narbad A. 2021. Meta-analysis of the parkinson's disease gut microbiome suggests alterations linked to intestinal inflammation. npj Parkinson's Disease 7:27.

Scheperjans F, Aho V, Pereira PAB, Koskinen K, Paulin L, Pekkonen E, et al. 2015. Gut microbiota are related to parkinson's disease and clinical phenotype. Movement Disorders 30:350-358.

Takahashi K, Nishiwaki H, Ito M, Iwaoka K, Takahashi K, Suzuki Y, et al. 2022. Altered gut microbiota in parkinson's disease patients with motor complications. Parkinsonism \& Related Disorders 95:11-17.

Tan A, Chong C, Teh C, Yap I, Loke M, Bowman J, et al. Unveiling the function of altered gut microbiota composition in parkinson's disease. In: Proceedings of the Movement Disorders, 2018, Vol. 33, S783-S784.

Tehrani R, Lyv MM, Kaveh R, Schnoor JL, Van Aken B. 2012. Biodegradation of mono-hydroxylated pcbs by burkholderia xenovorans. Biotechnol Lett 34:2247-2252.

Todd GD, Ruiz P, Mumtaz M, Wohlers D, Klotzbach JM, Diamond GL, et al. 2019. Toxicological profile for trichloroethylene (tce).

Toh TS, Chong CW, Lim SY, Bowman J, Cirstea M, Lin CH, et al. 2022. Gut microbiome in parkinson's disease: New insights from meta-analysis. Parkinsonism Relat Disord 94:1-9. 
Unger MM, Spiegel J, Dillmann K-U, Grundmann D, Philippeit H, Bürmann J, et al. 2016. Short chain fatty acids and gut microbiota differ between patients with parkinson's disease and age-matched controls. Parkinsonism \& Related Disorders 32:66-72.

USEPA. 2001. Sources, emission, and exposure for trichloroethylene (tce) and related compounds. .

Villumsen M, Aznar S, Pakkenberg B, Jess T, Brudek T. 2019. Inflammatory bowel disease increases the risk of parkinson's disease: A danish nationwide cohort study 1977-2014. Gut 68:18-24.

Wallen ZD, Appah M, Dean MN, Sesler CL, Factor SA, Molho E, et al. 2020. Characterizing dysbiosis of gut microbiome in pd: Evidence for overabundance of opportunistic pathogens. npj Parkinson's Disease 6.

Wallner A, King E, Ngonkeu ELM, Moulin L, Béna G. 2019. Genomic analyses of burkholderia cenocepacia reveal multiple species with differential host-adaptation to plants and humans. BMC Genomics 20:803803.

Wang H, Banerjee N, Liang Y, Wang G, Hoffman KL, Khan MF. 2021. Gut microbiome-host interactions in driving environmental pollutant trichloroethene-mediated autoimmunity. Toxicology and Applied Pharmacology 424:115597.

Wang H, Banerjee N, Liang Y, Wang G, Hoffman KL, Khan MF. 2021. Gut microbiome-host interactions in driving environmental pollutant trichloroethene-mediated autoimmunity. Toxicol Appl Pharmacol 424:115597.

Zapała B, Stefura T, Wójcik-Pędziwiatr M, Kabut R, Bałajewicz-Nowak M, Milewicz T, et al. 2021. Differences in the composition of gut microbiota between patients with parkinson's disease and healthy controls: A cohort study. J Clin Med; doi: 10.3390/jcm10235698.

Zhang F, Yue L, Fang X, Wang G, Li C, Sun X, et al. 2020. Altered gut microbiota in parkinson's disease patients/healthy spouses and its association with clinical features. Parkinsonism \& Related Disorders 81:84-88. 